\title{
The Impact of Blockchain on the Economic Development of Zhoushan Island Homestay and Its Prospect
}

Yingyan $\mathrm{Xu}^{*}$

Zhejiang Wanli University, Ningbo 315000, Zhejiang, China. E-mail: 1136875317@qq.com

Abstract: Since the 20th century, Zhoushan Island has gradually become a powerful country in terms of the location of the island and the development of its ocean tourism industry. However, there are some problems in Zhoushan's B \& B industry, which is still in the initial stage of development. "Blockchain + B \& B" takes advantage of blockchain to further enhance the economic vitality of Zhoushan B \& B. Of course, there are also deficiencies in the development of blockchain, which should be used reasonably.

Keywords: Blockchain; Homestay; Impact; Outlook

\section{Introduction of blockchain}

Since blockchain was first proposed, as a new concept in the field of Internet and computer, blockchain has attracted people's attention, and it is considered to be the successor of mainframe computer, personal computer and Internet. It is the fifth subversive computing paradigm after mobile social networking. Blockchain is a new application mode of computer technology such as distributed data storage, point-to-point transmission, consensus mechanism and encryption algorithm ${ }^{[1]}$. Compared with the traditional Internet, blockchain does not rely on the third-party management organization. In addition to the blockchain itself, each node realizes information self verification and management through distributed accounting and storage, and has the characteristics of decentralization. If someone tries to modify the network data, he must hold $51 \%$ of all the data, which is difficult to achieve, which reflects its security.

Blockchain has great application value in many fields. It can bring profound changes to finance, public service and public welfare.

\section{Economic development of Zhoushan Island B \& B}

B \& B is a kind of form for tourists to rent the private residence of B \& B owners. Tourists can enjoy the beautiful scenery and shopping around the B \& B. Driven by the market demand, the quantity and economic benefits of B \& B in China are developing vigorously ${ }^{[2]}$. Since 1990s, Zhoushan archipelago has won the favor of many tourists due to its unique geographical location, the policy guidance of accelerating, the construction of marine power and the great development of marine culture. With the continuous development of the B \& B industry, the island B \& B industry has gradually become the accommodation form of island tourism ${ }^{[3]}$.

\section{The impact of blockchain on the economic development of Zhoushan Is- land homestay}

This is an open-access article distributed under the terms of the Creative Commons Attribution Non-Commercial License

(http://creativecommons.org/licenses/by-nc/4.0/), which permits unrestricted non-commercial use, distribution, and reproduction in any medium,

provided the original work is properly cited. 
"Blockchain + B \& B" with the advantages of blockchain, is very important to guarantee the information of B \& B, standardize the management mechanism of B \& B, and improve the accommodation experience, which is very important to further enhance the economic vitality of Zhoushan B \& B, make good use of Zhoushan's rich marine resources, and build Zhoushan B \& B into a brand and certain model ${ }^{[4]}$.

\subsection{To ensure the information security of $B$ \& $B$}

The continuous development of Zhoushan B \& B has increased the complexity of the B \& B market. Blockchain has time stamp, data update which requires a specific number of nodes authentication. It has the technical advantage of tamper proof. The use of blockchain to a certain extent can avoid the possibility of brushing orders, and delete bad comments $^{[5]}$. On the one hand, tourists can choose B \& B according to their comments, which will greatly reduce the economic cost of finding B \& B and improve the efficiency of finding suitable B \& B. On the other hand, there will be a healthy competition between the host of B \& B. They will spend more time on improving the quality of B \& B.

\subsection{The management mechanism of $B$ \& $B$ has been standardized}

As Zhoushan B \& B is becoming more and more popular, the relevant departments are not able to rely solely on human resources management mechanism. The integration of block chain to improve the B \& B management mechanism meets the needs of consumers. With the help of blockchain, relevant departments store invoices and transaction information through blockchain to retain evidence for handling B \& B disputes. Through opening user channels on the Internet, consumers participate in the decision-making of relevant departments through these channels, which enables the decision-making level of relevant departments to listen to the public opinion to the maximum extent. In addition, autonomous organizations can also participate in the management process through blockchain ${ }^{[6]}$.

\subsection{To improved accommodation experience}

As a new technology, blockchain, combined with specific application scenarios, can release market demand. Blockchain can be skillfully combined with a variety of service scenarios. For example, with the help of blockchain, the risk of luggage loss will be minimized in the process of luggage transportation.

\section{The prospect of blockchain to the economic development of Zhoushan Island homestay}

There are still many deficiencies in the development of Zhoushan B \& B. Due to the lack of financial and technical support, there are some B \& B operators who build Zhoushan B \& B at will, which are not welcomed by consumers after they are put into the market. In order to reduce the losses, these B \& B operators blindly reduce the B \& B prices ${ }^{[7]}$. The B \& B market is mixed, which leaves a bad impression on consumers. Some island homestay's road, water and electricity facilities, safety supervision system and other infrastructure are not perfect, which brings inconvenience to consumers.

The only way to further develop Zhoushan B \& B economy and build smart B \& B is to combine blockchain technology.

\subsection{To establish the development goal of island B \& B}

Through the blockchain technology, relevant departments have advanced positioning for Zhoushan Island homestay. The personalized planning of Zhoushan Island B \& B makes the host provide perfect service to tourists based on the needs of tourists, so as to make the host get more profits.

\subsection{To establish public service platform for famous hotels on the island}

It has established an information service platform leading to microblog, Wechat and other apps, providing various services such as tourism map and VR. It not only creates diversified information services for tourists, but also provides a communication platform for tourists. 


\subsection{To improve AI services}

In recent years, artificial intelligence technology is very popular, and various fields and industries are more or less applied to artificial intelligence. For the B \& B industry, artificial intelligence will also play an irreplaceable role. The smart contract will be executed automatically according to the agreement reached between the main bodies. The self energy contract will lock the consumption funds of tourists. Only when both parties confirm that the service is completed, the locked funds will be transferred to the host's account. This kind of contract can guarantee fairness and order. Before staying in the B \& B, consumers write the accommodation expectation into the contract, and execute the smart contract after the condition is triggered. The host optimizes the service content and quality: provide intelligent housekeeper for tourists, report the time intelligently, and exchange weather conditions with tourists ${ }^{[8,9]}$.

\subsection{To innovate marketing methods}

A successful marketing model can greatly attract tourists and increase revenue. In the aspect of B \& B marketing, the marketing channels can be broadened through experience marketing and online group buying mode, and the influence can be expanded by means of crowdfunding and cultural promotion ${ }^{[10]}$.

\section{Conclusion}

As an important part of the tertiary industry in Zhoushan, Zhoushan Island homestay industry has its shortcomings in the development process, which can be better improved by using the advantages of blockchain security. With the continuous development of the blockchain, Zhoushan B \& B has been able to produce greater capacity.

Nevertheless, we also need to realize that the blockchain itself is still constrained by the appropriate conditions, and needs to be further strengthened in terms of technology, economy and so on. In the process of combining the B \& B industry with the blockchain, we should be cautious.

\section{References}

1. Chen M. Evaluation on the development of island homestay tourism resources in Zhoushan and the protection strategies. Ocean Development and Management 2017; 34(6): 23-27.

2. Wang C. Research on insurance system architecture and key technology based on blockchain [PhD thesis]. Beijing: China Academy of Railway Sciences; 2017.

3. Xu X, Li H, Ma M. Blockchain + tourism services: Innovation prospects and potential challenges. Chinese Public Administration 2019; (4): 53-57, 78.

4. Huang Q. The challenge of blockchain high-tech application in tourism Internet. Computer Engineering \& Software 2019; 40(12): 90-96.

5. Tao Y. Research on Zhoushan Island B \& B under the background of leisure fishery development [Master's thesis]. Zhejiang: Zhejiang Ocean University; 2019.

6. Jiang S. A case study of traditional village home stay and community participation in Tonglu County (in Chinese) [Master's thesis]. Zhejiang: Zhejiang Gongshang Universitys; 2015.

7. Chen M. On the excitation pattern and countermeasures of the island village homestay in the light of "Internet + " (in Chinese). Journal of Zhejiang Ocean University (Humanities Sciences) 2017; 34(2): 56-60, 71.

8. Yang X. Research on credit and trust mechanism in electronic business environment [PhD thesis]. Beijing: University of international business and Economics; 2016.

9. Gu J. To build a aggregation of island homestay in the era of shared economy: A case study of Zhejiang Zhoushan archipelago new area. Special Zone Economy 2017; (11): 85-87.

10. Yang H, Chen X. A case study of Shengsi County in Zhejiang Province on the development of island homestay industry (in Chinese). Rural Economy and Science-Technology 2016; 27(21): 92-94. 